\title{
Dynamical black hole in a bouncing universe
}

\author{
Daniela Pérez* \\ Instituto Argentino de Radioastronomía (IAR, CONICET/CIC/UNLP), \\ C.C.5, (1894) Villa Elisa, Buenos Aires, Argentina \\ Santiago E. Perez Bergliaffa \\ Departamento de Física Teórica, Instituto de Física, \\ Universidade do Estado de Rio de Janeiro, CEP 20550-013, Rio de Janeiro, Brazil \\ Gustavo E. Romerd \\ Instituto Argentino de Radioastronomía (IAR, CONICET/CIC/UNLP), \\ C.C.5, (1894) Villa Elisa, Buenos Aires, Argentina
}

(Dated: March 2, 2021)

\begin{abstract}
We analyze the causal structure of McVittie spacetime for a classical bouncing cosmological model. In particular, we compute the trapping horizons of the metric and integrate the trajectories of radial null geodesics before, during, and after the bounce takes place. In the contracting phase up to the occurrence of the bounce, a dynamical black hole is present. When the universe reaches a certain minimum scale, the trapping horizons disappear and the black hole ceases to exist. After the bounce, the central weak singularity becomes naked. In the expanding phase, for large positive values of the cosmic time, the behaviour of null geodesics indicates that the solution contains a black hole. These results suggest that neither a contracting nor an expanding universe can accommodate a black hole at all times.
\end{abstract}

\section{INTRODUCTION}

Our current understanding of the evolution of the Universe is expressed in the so-called $\Lambda \mathrm{CDM}$ model that includes gravity as described by the Einstein's field equations with a cosmological term, the standard model of particle physics, a component of cold dark matter, and a hot initial phase. This model is frequently complemented with an inflationary stage that would have occurred immediately before the grand unification epoch. Inflation requires additional physics beyond the standard model. With or without inflation the standard cosmological model is singular [1]. An initial cosmological singularity is a very undesirable feature that points out an irreparable deficiency in the representation of the underlying physical processes 2 . Bouncing cosmologies try to offer an alternative to overcome such problems.

There is a wide variety of proposals, either classical or quantum, for a cosmological bounce 3. In all these models the universe starts from a very diluted phase and proceeds to contract. The contraction then smoothly evolves into a bounce that leads to the current phase of expansion as described by the $\Lambda \mathrm{CDM}$ model. These models solve the problem of the initial cosmological singularity. As the cosmic fluid contracts most structure is erased and the universe becomes smooth [4. Black holes, however, might survive the bounce and play some role in the subsequent expanding universe [5-7]. Since a

\footnotetext{
* danielaperez@iar.unlp.edu.ar

† Also at Facultad de Ciencias Astronómicas y Geofísicas, Universidad Nacional de La Plata, Paseo del Bosque s/n, 1900 La Plata, Buenos Aires, Argentina
}

black hole is essentially a region of spacetime with particular curvature, the overall contraction and expansion of space during the bounce should have a global-to-local effect upon its horizons. The whole process is dynamical, and hence cannot be investigated using the standard static solutions.

An exact solution for a central inhomogeneity in a cosmological setting was first found by McVittie long ago. It is now clear that such a solution describes a black hole. McVittie solution has been investigated, so far, for standard prescriptions of the scale factor of the universe. In this work we extend the research to models that allow for a bounce. We discuss whether the solution includes a black hole before the bounce and what happens with the horizons along the cosmological history of a bouncing universe. The results we have found, we hope, will help to obtain a better understanding of both the McVittie solution and the fate of a dynamical black hole through a cosmic bounce.

\section{DYNAMICAL SPACETIMES}

\section{A. McVittie spacetime}

In 1933, McVittie 8] discovered an exact solution of Einstein's field equations that describes an inhomogeneity embedded in a Friedmann-Lemaitre-RobertsonWalker (FLRW) cosmological background. In isotropic coordi- 
nates $(t, r, \theta, \phi)$ its line element takes the form:

$$
\begin{aligned}
d s^{2} & =-\frac{\left(1-\frac{m_{0}}{2 r a(t)}\right)^{2}}{\left(1+\frac{m_{0}}{2 r a(t)}\right)^{2}} d t^{2}+a^{2}(t)\left(1+\frac{m_{0}}{2 r a(t)}\right)^{4} \\
& \times\left[d r^{2}+r^{2}\left(d \theta^{2}+\sin ^{2} \theta d \phi^{2}\right)\right] .
\end{aligned}
$$

Here, $a(t)$ is the scale factor of the background cosmological model and $m_{0}$ is a non-negative constant. Setting $a(t) \equiv 1$, Eq. (1) reduces to the Schwarzschild line element in isotropic coordinates, and in the limit $m_{0} \rightarrow 0$ the FLRW metric is recovered. For the upcoming discussion, it is convenient to express Eq. (1) in terms of $R$, the areal radius coordinate [9]:

$$
R \equiv a(t) r\left(1+\frac{m_{0}}{2 r a(t)}\right)^{2} .
$$

Using this expression, the McVittie line element can be written as

$$
\begin{aligned}
d s^{2} & =-f(t, R) d t^{2}-\frac{2 H(t) R}{\sqrt{1-2 m_{0} / R}} d t d R+\frac{d R^{2}}{1-2 m_{0} / R} \\
& +R^{2}\left(d \theta^{2}+\sin ^{2} \theta d \phi^{2}\right),
\end{aligned}
$$

where

$$
f(t, R) \equiv 1-2 m_{0} / R-H(t)^{2} R^{2} .
$$

Here, $H(t) \equiv \dot{a}(t) / a(t)$ is the Hubble factor corresponding to the background cosmological model. There are two key assumptions on which McVittie's solution is based. The first one is that the matter represented in the field equations is described by a perfect fluid with density $\rho$ and isotropic pressure $p$. The second one is that the fluid is at rest with respect to the chosen reference frame. There are no additional hypothesis regarding the properties of the matter that sources the geometry. In particular, an equation of state, for instance of the form $p=p(\rho)$, is not assumed. The relation between $\rho$ and $p$ is obtained a posteriori by solving Einstein field equations when the scale function $a(t)$ is specified. For further details on the derivation of the McVittie solution see Refs. [10 12].

There has been a long debate in the literature about the physical interpretation of the McVittie spacetime, focused mainly on deciding whether the solution characterizes a black hole in an expanding universe or not. A recent series of works [11-15] has been crucial to establish that the McVittie metric represents a dynamical black hole embedded in a cosmological background. The details of the solution and its possible analytical extension depend of the behaviour of $H(t)$ for $t \rightarrow \infty$ [15. The solution displays a curvature singularity at $R=2 m_{0}$ for finite values of $t$, as evidenced by the Ricci scalar, given by:

$$
\mathcal{R}=12 H^{2}+\frac{6 \dot{H}}{\sqrt{1-\frac{2 m_{0}}{R}}} .
$$

The singularity is spacelike and, as shown by Nolan [13], gravitationally weak [16].

The key feature associated with black holes is the presence of an event horizon, i.e. a boundary between two regions of spacetime that are causally disconnected. Events inside the black hole are separated from events in the global external future of spacetime. In dynamical spacetimes, in order to identify the event horizon, we would need to know the entire spacetime manifold to future infinity, which is impossible if the metric is not completely known.

In order to determine whether a black hole is embedded in a dynamical background, a full analysis of the causal structure of the spacetime is necessary. This includes studying the existence of trapping horizons, the determination of regular trapped and anti-trapped regions, and the computation of the trajectories of ingoing and outgoing radial null geodesics. In what follows, we briefly review the main results of Refs. 11 15 that prove the assertion that the McVittie spacetime describes a black hole in a cosmological environment.

The trapping horizons of a spacetime are defined as the surfaces where null geodesics change their focusing properties [17]. Mathematically, this kind of horizon is determined by the condition

$$
\theta_{\text {in }} \theta_{\text {out }}=0,
$$

where $\theta_{\text {in }}$ stands for the expansion of ingoing radial null geodesics while $\theta_{\text {out }}$ denotes the expansion of outgoing radial null geodesics, respectively. Regions where $\theta_{\text {in }} \theta_{\text {out }}<0$ are called regular. In the opposite case, $\theta_{\text {in }} \theta_{\text {out }}>0$, the region is called anti-trapped if $\theta_{\text {in }}>0$ and $\theta_{\text {out }}>0$, and trapped if $\theta_{\text {in }}<0$ and $\theta_{\text {out }}<0$. In this section, we are only considering expanding spacetimes, i.e. $H(t)>0$. The trapping horizons for the McVittie metric occur for $\theta_{\text {in }}=0$, and null outgoing geodesics are always expanding $\left(\theta_{\text {out }}>0\right)[14,15$.

We show in Figures 1 and 2 the location of the trapping horizons in the McVittie spacetime for the $\Lambda \mathrm{CDM}$ model [18] and for a dust background. In the first case, the corresponding Hubble factor is $H(t)=H_{0} \operatorname{coth}\left(3 / 2 H_{0} t\right)$, where $H_{0}$ is the Hubble constant, while in the second case $\mathrm{H}(\mathrm{t})=2 / 3 \mathrm{t}$ [15]. The essential difference between these two cosmological models is the asymptotic behaviour of the Hubble factor in the future: $H(t) \rightarrow H_{0}$ for $t \rightarrow \infty$ in the $\Lambda \mathrm{CDM}$ model whereas $H(t) \rightarrow 0$ for $t \rightarrow \infty$ in a dust-dominated background.

There is a moment in time, denoted $t_{\star}$, when just one trapping horizon exists. It can be computed by solving the following equation:

$$
m_{0} H\left(t_{\star}\right)=\frac{1}{3 \sqrt{3}} .
$$

If the cosmological background corresponds to the $\Lambda$ CDM model,

$$
t_{\star \Lambda \mathrm{CDM}}=\frac{2}{3 H_{0}} \operatorname{arcoth}\left(\frac{1}{3 \sqrt{3} m_{0} H_{0}}\right),
$$




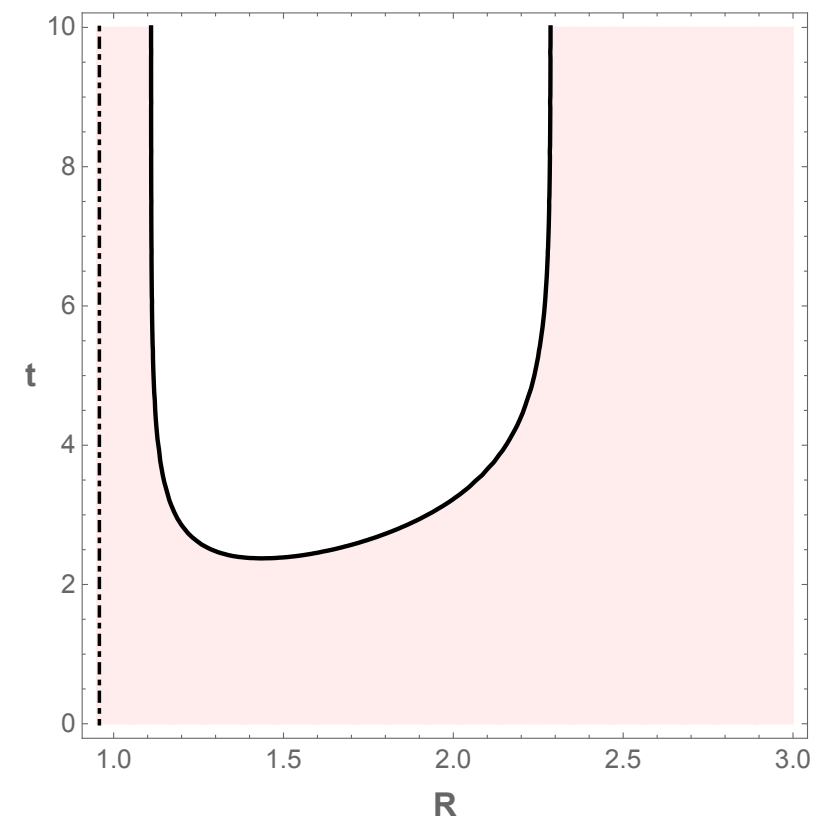

FIG. 1. The black line indicates the location of the trapping horizons in McVittie spacetime for the $\Lambda$ CDM model. The white zone corresponds to the regular region while the light pink corresponds to the anti-trapped region, respectively. The dot dashed line denotes the location of the singularity. Here, we fixed $m_{0}=0.479$ and $H_{0}=1 / 3$ as an example.

and when the cosmological background is dustdominated,

$$
t_{\star \text { dust }}=2 \sqrt{3} m_{0} .
$$

Inspection of Figures 1 and 2 reveals that when $t>t_{\star}$ there are two trapping horizons: an inner (denoted $R_{-}$) and an outer one (denoted $R_{+}$), such that $R_{+}>R_{-}$. No trapping horizons are present for $t<t_{\star}$. In both figures, the regular region of the spacetime is indicated in white while the anti-trapped region is painted in light pink. The dot-dashed curve indicates the location of the singularity.

Due to spherical symmetry, the equation for the ingoing and outgoing radial geodesics can be derived by setting $d \theta=d \phi=0$ in $d s^{2}=0$, thus obtaining

$$
\frac{d R}{d t}=\sqrt{1-2 m_{0} / R}\left(H R \pm \sqrt{1-2 m_{0} / R}\right),
$$

where the "-" ("+") corresponds to the ingoing (outgoing) case. We see that $d R / d t>0$ for the outgoing branch, i.e, such geodesics are always diverging. This result is consistent with the fact that $\theta_{\text {out }}>0$, as mentioned above. We plot these trajectories in Figures 3 and 4.

As seen from Eq. 10, only radial ingoing null geodesics have a turning point, defined by $d R / d t=0$, and specified by those values of the coordinates $R$ and $t$ that obey the following equation:

$$
\tilde{f}(t, R)=H(t)^{2} R^{3}-R+2 m_{0}=0 .
$$

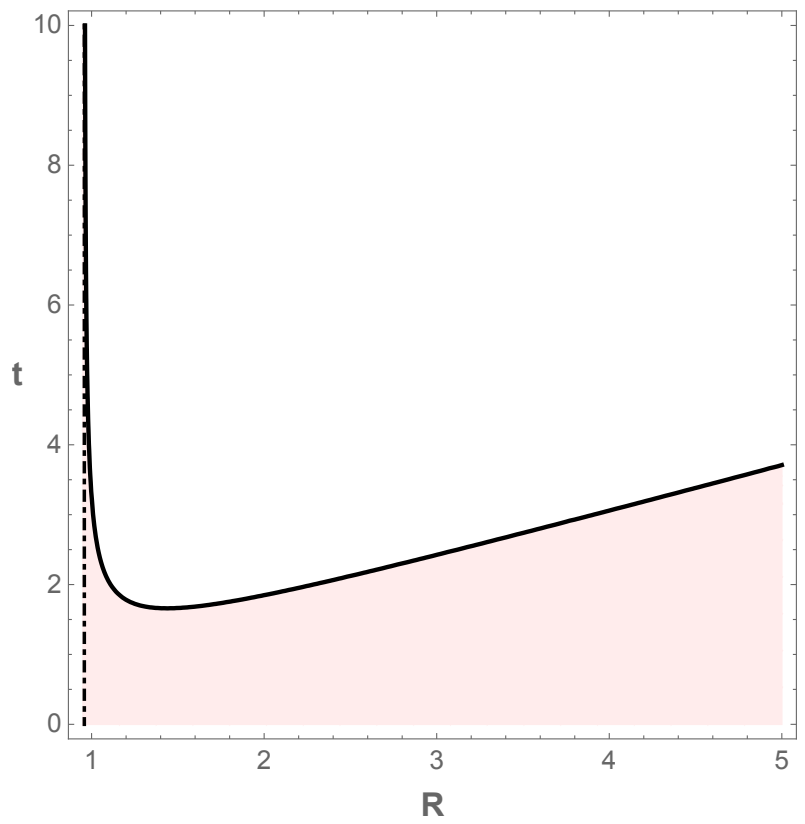

FIG. 2. The black line indicates the location of the trapping horizons in McVittie spacetime for a dust background model. The white zone corresponds to the regular region while the light pink corresponds to the anti-trapped region, respectively. The dot dashed line denotes the location of the singularity. Here, we fixed $m_{0}=0.479$ as an example.

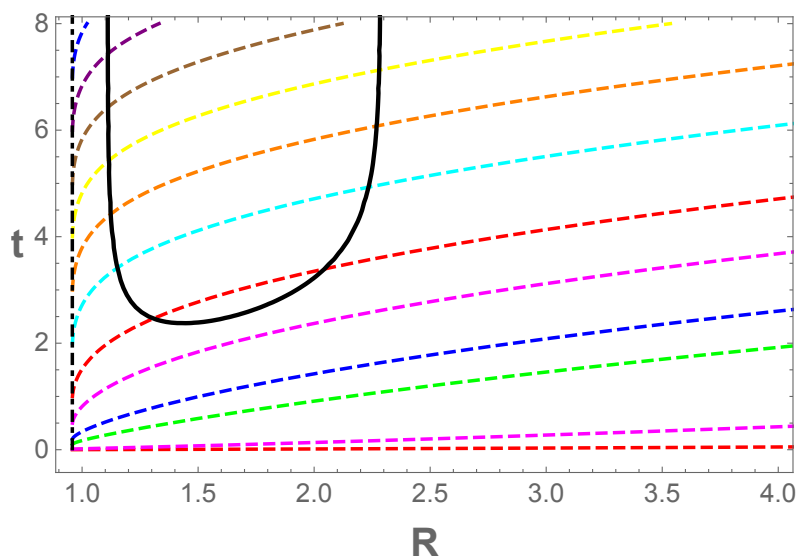

FIG. 3. Radial outgoing null geodesics in McVittie spacetime for the $\Lambda$ CDM model. The black line indicates the location of the trapping horizons and the dot-dashed line denotes the singular surface $R=2 m_{0}$. Here, we fixed $m_{0}=0.479$ and $H_{0}=1 / 3$ as an example.

Clearly, Eq. (11) is equivalent to $\theta_{\text {in }}=0$. From Figures 5 and 6 we see that the radial ingoing geodesics are expanding in the anti-trapped region $\left(\theta_{\text {in }}>0\right)$ and they converge in the regular zone $\left(\theta_{\text {in }}<0\right)$. When they cross the trapping horizon $\left(\theta_{\text {in }}=0\right.$ or equivalently $\left.\tilde{f}(t, R)=0\right)$, their convergence changes sign.

We emphasise that trapping horizons are not equivalent to event horizons in the context of dynamical space- 


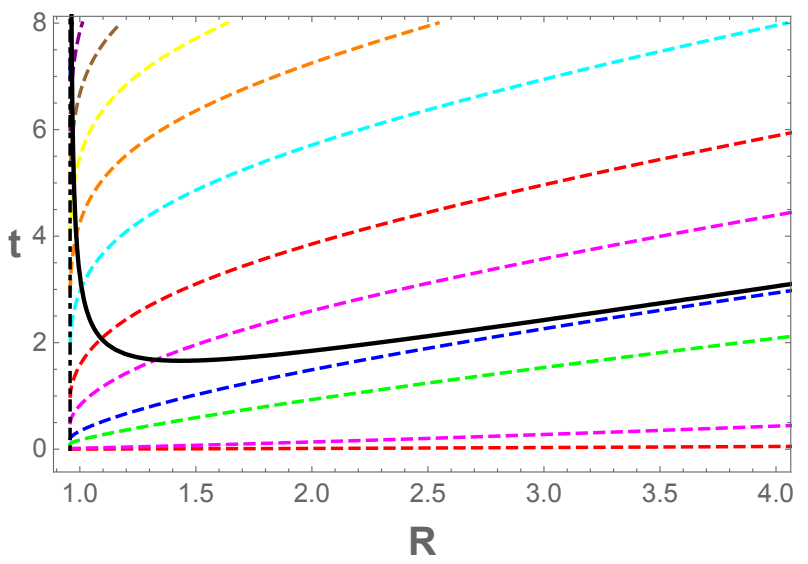

FIG. 4. Radial outgoing null geodesics in McVittie spacetime for a dust cosmological background. The black line indicates the location of the trapping horizons and the dot-dashed line denotes the singular surface $R=2 m_{0}$. Here, we fixed $m_{0}=$ 0.479 as an example.

times. As shown in Figures 3 and 4 , some outgoing geodesics cross both $R_{-}$and $R_{+}$. In Figure 5 , the green, yellow, brown, and blue geodesics cross $R_{+}$and enter the regular region of the spacetime; as time goes by, they get closer and closer to the surface $R_{-}$. We stress that for finite values of the time coordinate, $R_{-}$is just a trapping horizon. Only in the limit $t \rightarrow \infty, R_{-}$becomes an event horizon and outgoing geodesics cannot travel out of the black hole. In fact, Kaloper and collaborators [14] proved that, under certain assumptions, the analysis of the behavior of ingoing null geodesics in the limit $t \rightarrow \infty$ reveals the presence of an event horizon.

Assuming that $H(t \rightarrow \infty) \rightarrow H_{0}=$ constant, two surfaces are particularly relevant in this spacetime [14:

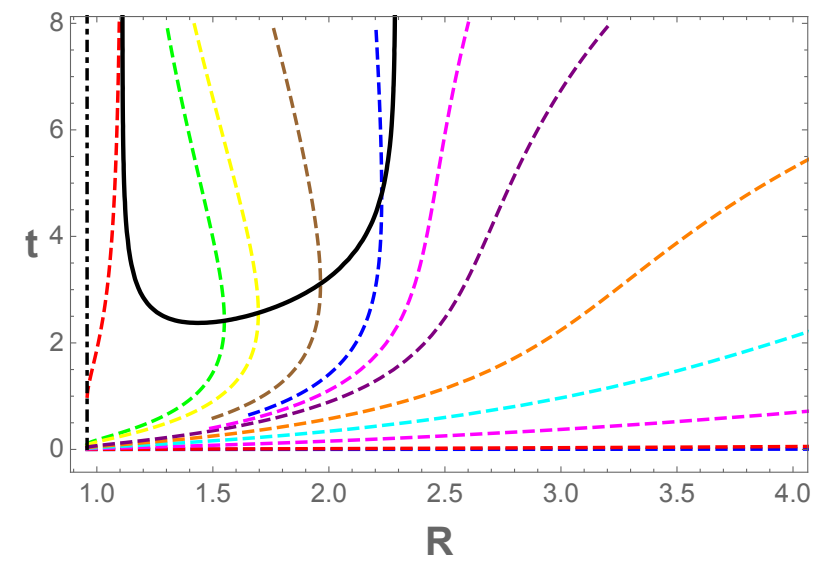

FIG. 5. Radial ingoing null geodesics in McVittie spacetime for the $\Lambda \mathrm{CDM}$ model. The black line indicates the location of the trapping horizons and the dot-dashed curve denotes the singular surface $R=2 m_{0}$. Here, we fixed $m_{0}=0.479$ and $H_{0}=1 / 3$ as an example.
- A null surface at $R=R_{-}, t \rightarrow \infty$, where $R_{-}$is the smaller positive root of $\tilde{f}(t \rightarrow \infty, R)=H_{0}^{2} R^{3}-R+$ $2 m_{0}=0$.

- A null surface at $R=R_{+}, t \rightarrow \infty$, where $R_{+}$is the larger positive root of $\tilde{f}(t \rightarrow \infty, R)=H_{0}^{2} R^{3}-R+$ $2 m_{0}=0$.

If the null energy condition is satisfied, it was demonstrated in Ref. 14] that:

1. Null ingoing geodesics in the regular region of the spacetime cross the surface $R=R_{-}, t \rightarrow \infty$ at a finite value of the affine parameter.

2. Such surface is regular (i.e. all the squared curvature invariants constructed with the Riemann tensor and its contractions are finite on it) [19].

3. Once the geodesics transverse the surface $R=R_{-}$, $t=t_{\infty}$, they are in a trapped region since $\theta_{\text {in }} \theta_{\text {out }}>$ 0 there.

Consequently, those geodesics that go through the surface $R=R_{-}, t \rightarrow \infty$, will not cross it again in the opposite direction. This is precisely the situation in the presence of an event horizon. The conclusion is that the solution represents a black hole with an horizon at $R=R_{-}$ at large times.

Using a similar procedure as the one describe above, Lake and Abdelqader (see Appendix D in 15]) also demonstrated that in the case $H(t \rightarrow \infty) \rightarrow H_{0}=0$ (for instance, the dust-dominated background previously discussed), the McVittie metric represents a black hole in the future.

Having presented the main features of the McVittie solution, we introduce in the next section the cosmological background model that we adopt in this work.

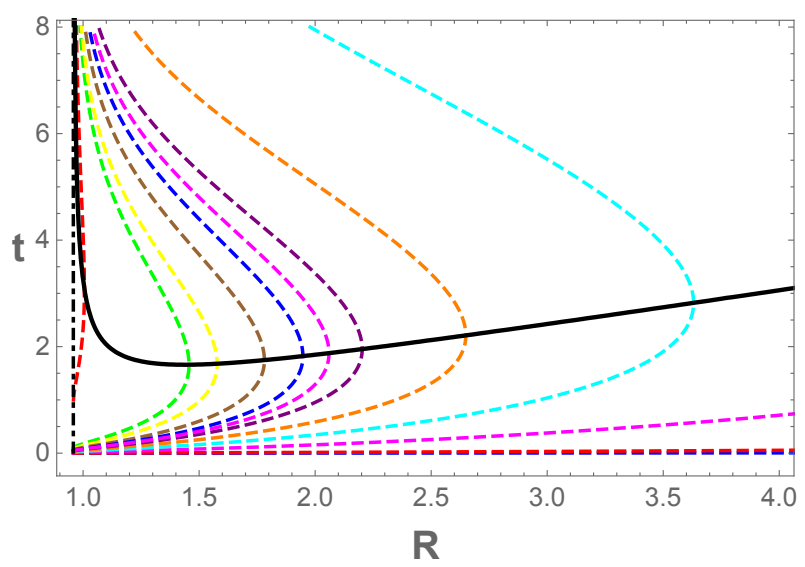

FIG. 6. Radial ingoing null geodesics in McVittie spacetime for a dust cosmological background. The black line indicates the location of the trapping horizons and the dot-dashed curve denotes the singular surface $R=2 m_{0}$. Here, we fixed $m_{0}=$ 0.479 as an example. 


\section{SCALE FACTOR FOR A BOUNCING COSMOLOGICAL MODEL}

Cosmological models that display a bounce solve by construction the initial singularity problem, as well as the horizon and flatness problems of the standard cosmological model 20]. Such models can also produce primordial cosmological perturbations from vacuum fluctuations, with an almost scale-invariant spectrum [21, and can be viewed either as an alternative or a complement to inflation (see for instance Ref. 22]). Typically, models with a bounce join a contracting phase, in which the Universe was very large and almost flat initially, to a subsequent expanding phase. The bounce can be either generated classically (see e.g. Refs. 23 25]), or by quantum effects (see e.g. Refs. 221, 26, 28). Since our aim is to investigate the effects of the bounce on the McVittie solution, with no intention at this stage to build a complete cosmological model, our choice of the regular model will be guided by simplicity. The expression for the scale factor we adopt as background in our work, given by

$$
a(t)=a_{0}\left[1+\left(\frac{t}{t_{0}}\right)^{2}\right]^{1 / 3}
$$

was found in 29] by considering quantum corrections to the classical evolution of the scale factor. The corrections were obtained by solving the Wheeler-deWitt equation in the presence of a single perfect fluid, in the framework of the de Broglie-Bohm quantum theory [30. Other quantization methods yield the same evolution for the scale factor, see Refs. 3133 . Notice that the scale factor reduces to that of dust for $t>>t_{0}$, and leads to an evolution that is dominated near the bounce by an effective fluid with negative energy density that scales as $a^{-6}$, as can be seen from Friedman's equation 34 .

\section{MCVITTIE SPACETIME IN A BOUNCING COSMOLOGICAL MODEL}

Our goal is to compute and analyze the causal structure of the McVittie spacetime in a classical cosmological bouncing model. As we discussed above, under certain assumptions, the McVittie metric represents a black hole. In what follows, we will examine how this solution behaves before, during, and after the bounce, and whether a black hole is present in any of these stages of cosmological evolution.

\section{A. Trapping horizons and null geodesics}

We begin by computing the trapping horizons using Eq. (11) and the Hubble factor

$$
H(\tilde{t})=\frac{2}{3 t_{0}} \frac{\tilde{t}}{1+\tilde{t}^{2}},
$$

in the time interval $-\infty<\tilde{t}<+\infty$ [35]. In Eq. 113], the new variable $\tilde{t}$ is defined as $\tilde{t} \equiv t / t_{0}$. In what follows, to simplify the notation, we replace $\tilde{t}$ by $t$. We rewrite Eq. (11) recovering the corresponding units:

$$
\frac{H(t)^{2}}{c^{2}} R^{3}-R+2 \frac{G m_{0}}{c^{2}}=0 .
$$

Defining a new dimensionless variable as $x \equiv$ $R /\left(G m_{0} / c^{2}\right)$, the latter equation takes the form

$$
\alpha^{2} H(t)^{2} x^{3}-x+2=0,
$$

where $\alpha=G m_{0} / c^{3}$. In order to solve this cubic equation, we express 15 as 36 ]

$$
\alpha^{2} H(t)^{2} x^{3}-3 \alpha^{2} H(t)^{2} \delta^{2} x+2=0 .
$$

Here, $\delta^{2} \equiv 1 /\left(3 \alpha^{2} H(t)^{2}\right)$. By performing the change of variables $x=2 \delta \sin \phi$, and after some simple algebraic manipulations, Eq. (16) becomes

$$
\begin{aligned}
2 \alpha^{2} H(t)^{2} \delta^{3}\left(4 \sin ^{3} \phi-3 \sin \phi\right)+2 & =0 \\
-\alpha^{2} H(t)^{2} \delta^{3} \sin 3 \phi+1 & =0 .
\end{aligned}
$$

Trapping horizons exist only if $0<\sin 3 \phi<1$. In particular, let us focus on the case where the radial coordinate of the trapping horizons coincides; this occurs for $\sin 3 \phi=1$, which in terms of Eq. 17 takes the form

$$
3 \sqrt{3} \frac{G m_{0}}{c^{3}} H(t)=1, \Rightarrow t^{2}-2 \sqrt{3} \frac{G m_{0}}{c^{3} t_{0}} t+1=0 .
$$

The solutions for 18 are

$$
t=\sqrt{3} \frac{G m_{0}}{c^{3} t_{0}} \pm \sqrt{\left(\sqrt{3} \frac{G m_{0}}{c^{3} t_{0}}\right)^{2}-1}
$$

Thus, depending on the value of the mass of the central source $m_{0}$ and $t_{0}$, which fixes the time scale of the bounce, we have the following three cases [37]:

1. If $\frac{\sqrt{3} G m_{0}}{c^{3}}>t_{0}$, then there are two values of $t$ where $X_{-} \stackrel{c^{3}}{=} X_{+}$.

2. If $\frac{\sqrt{3} G m_{0}}{c^{3}}=t_{0}$, then there is only one value of $t$ where $X_{-}=X_{+}$.

3. If $\frac{\sqrt{3} G m_{0}}{c^{3}}<t_{0}$, then there is no value of $t$ where $X_{-}=X_{+}$.

According to Frion and collaborators 38, $10^{3} t_{\text {Planck }}<$ $t_{0}<10^{40} t_{\text {Planck }}$, that is $t_{0}^{\min }=10^{-41} \mathrm{~s}<t_{0}<t_{0}^{\max }=$ $10^{-4} \mathrm{~s}$. We show in Figure 7 the range of allowed values for $m_{0}$ (in units of solar masses $M_{\odot}$ ) according to the three conditions described above: the black line indicates the condition $\sqrt{3} G m_{0} / c^{3}=t_{0}$. The light yellow zone corresponds to values of $m_{0}$ and $t_{0}$ such that $\sqrt{3} G m_{0} / c^{3}>t_{0}$, while the light red region satisfies the condition $\sqrt{3} G m_{0} / c^{3}<t_{0}$. 


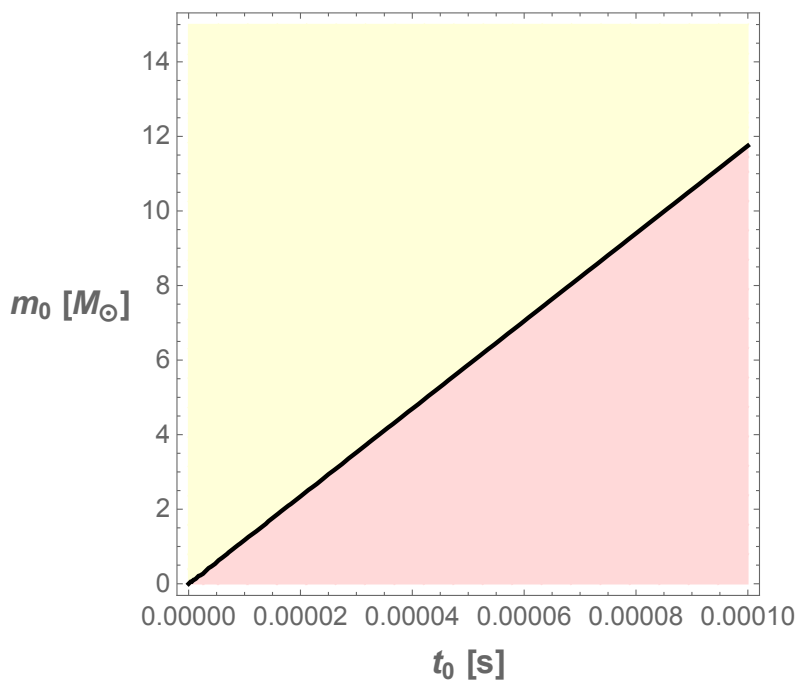

FIG. 7. The black line indicates the condition $\sqrt{3} G m_{0} / c^{3}=$ $t_{0}$. The light yellow zone corresponds to values of $m_{0}$ and $t_{0}$ such that $\sqrt{3} G m_{0} / c^{3}>t_{0}$ while the light red region satisfy the condition $\sqrt{3} G m_{0} / c^{3}<t_{0}$. Here, $m_{0}$ is in units of solar masses $M_{\odot}$.

From a physical point of view, it seems more attractive to consider a bounce time scale close to the allowed upper limit $\left(t_{0}^{\max }=10^{-4} \mathrm{~s}\right)$, that is, in the limit between a classical and quantum bounce. In such scenario, matter inhomogeneities that existed in the contracting phase might be able to go through the bounce and also be present in the expanding epoch. On the other hand, Carr and Kühnel [39] have recently showed that primordial black holes in the mass range $10 M_{\odot}<M<10^{2} M_{\odot}$ could be relevant to provide a fraction of the dark matter in the universe as well to explain the observed LIGO/Virgo coalescence events in the mass range $\mathcal{O}(10) M_{\odot}$. Therefore, in what follows we choose for $m_{0}$ and $t_{0}, m_{0}=50 M_{\odot}$ and $t_{0}=5 \times 10^{-5} \mathrm{~s}$. The structure of the trapping horizons that will be analysed in this work corresponds to case 1 .

The trapping horizons are plotted in Figure 8 8 the lower plot zooms into the region near the bounce. Very close to $t=0$, just before and after the bounce, there is a trapping horizon. This surface is absent, for instance, in McVittie spacetime for the dust-dominated background (see Figure 2). In Section VII, we argue that this horizon is a particular feature related to the presence of the bounce.

There are two additional trapping horizons for negative and positive values of the cosmic time, respectively. The shape of these surfaces is qualitatively similar compared to the trapping horizons in the McVittie solution for the dust background (see Figure 2): for $t>0$ there is a moment in time when a single trapping horizon begins to exist and immediately after an inner $X_{-}$and outer $X_{+}$ trapping horizons emerge. In the limit $t \rightarrow \infty, X_{-} \rightarrow 2$.

The second root of Eq. (11), namely $X_{+}$, is given by
40]

$$
X_{+}=\frac{c^{3}}{G m_{0}}\left[\frac{1}{H(t)} \cos \psi(t)-\frac{1}{\sqrt{3} H(t)} \sin \psi(t)\right]
$$

where $\sin 3 \psi(t)=3 \sqrt{3} G m_{0} H(t) / c^{3}$. For the Hubble factor given by Eq. (13), $X_{+} \rightarrow \infty$ when $t \rightarrow \pm \infty$, and thus $X_{+}$becomes a FLRW null infinity [14].

The shape of the curve of the horizons is symmetric with respect to the axis $t=0$. In the limit $t \rightarrow-\infty$, $X_{-} \rightarrow 2$. As we approach the bounce, $X_{-}$increases while $X_{+}$decreases up to they merge. The symmetry for the trapping horizons with respect to $t=0$ is rooted in the equation that defines these surfaces (see Eq. 111) or (15)), which is quadratic in the Hubble factor.

The white zones in Figure 8 indicate the regular regions of the spacetime $\left(\theta_{\text {in }} \theta_{\text {out }}<0\right)$, the light pink zone corresponds to the anti-trapped region $\left(\theta_{\text {in }} \theta_{\text {out }}>0\right.$, being $\theta_{\text {in }}>0$ and $\left.\theta_{\text {out }}>0\right)$, and the light blue zone marks the trapped region $\left(\theta_{\text {in }} \theta_{\text {out }}>0\right.$, being $\theta_{\text {in }}<0$ and $\left.\theta_{\text {out }}<0\right)$. The dot-dashed curve marks the location of the singularity $x=2$ for $t$ finite.

We also compute the trajectories of ingoing and outgoing radial null geodesics by integrating Eq. (10). The behaviour of the geodesics changes before and after the bounce:

- Outgoing null geodesics are always expanding $\left((d x / d t)_{\text {out }}>0\right)$ for $t>0$, as can be seen in Figure 9. Radial ingoing geodesics expand in the antitrapped region until they cross the trapping horizon; once in the regular region of the spacetime $(d x / d t)_{\text {in }}<0$, they all seem to tend asymptotically to the surface $X_{-}=2, t=\infty$, as shown in Figure 10. Below, we will provide a more detail analysis of the properties of the ingoing null geodesics in the limit $t \rightarrow \infty$, which is essential to establish whether a black hole is present.

- Ingoing and outgoing radial null geodesics reverse their character for $t<0$. Now, all ingoing trajectories are expanding to the past of the bounce (for increasing negative values of the $t$ coordinate). This is in Figure 10. Outgoing null geodesics converge in the trapped region. To the past of these geodesics, they seem to come from the surface $X_{-}=2$, $t=-\infty$ (see Fig. 9p. In Section VI we offer a possible interpretation of the McVittie solution before the bounce takes place.

In Figures 11 and 12 we offer a close up of the region near the bounce showing the behaviour of the outgoing and ingoing null geodesics. Some null geodesics (painted in blue and red in Figure 11) start at the remote past near $x=2$, go through the bounce and expand getting away from the central inhomogeneity. The same occurs for some ingoing geodesics. This does not happen in any McVittie model without a bounce analyzed so far in the literature; in those spacetimes the singularity at 

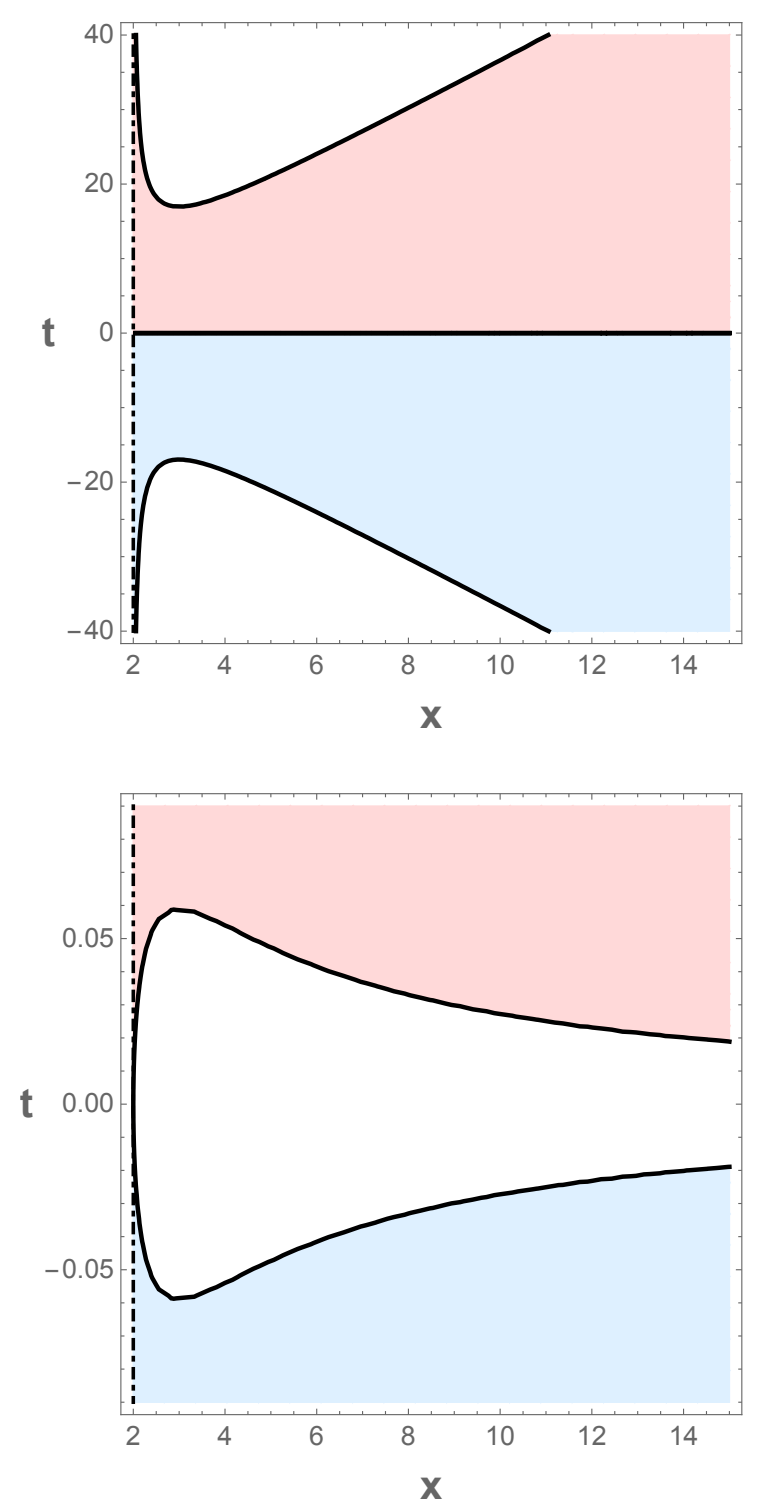

FIG. 8. The black lines indicate the location of the trapping horizons. The white zones correspond the regular regions, the light pink zone indicates the anti-trapped region and the light blue zone denotes the trapped region. The dot dashed curve denotes the location of the singularity. The lower plot zooms into the region near the bounce. Here, $m_{0}=50 M_{\odot}$, and $t_{0}=5 \times 10^{-5} \mathrm{~s}$

$x=2 m_{0}, t$ finite, lies in the causal past of all events, and thus it is regarded as a cosmological big bang singularity [14. In the present model, the existence of a cosmological bounce renders only some spacetime trajectories geodesically incomplete.

The equation for the ingoing radial geodesics in terms of the Hubble factor 13 takes the form

$$
\left.\frac{d x}{d t}\right|_{\text {in }}=\frac{1}{\alpha} \sqrt{1-2 / x}\left(\frac{2 \alpha}{3} \frac{t}{1+t^{2}} x-\sqrt{1-2 / x}\right),
$$

where $\alpha=G m_{0} /\left(c^{3} t_{0}\right)$. Before the bounce, the Hubble factor can be rewritten as $H(t)=-2 t /\left(3 t_{0}\left(1+t^{2}\right)\right)$ where $t>0$. Replacing into 21 :

$$
\begin{aligned}
\left.\frac{d x}{d t}\right|_{\text {in }} & =-\left[\frac{1}{\alpha} \sqrt{1-2 / x}\left(\frac{2 \alpha}{3} \frac{t}{1+t^{2}} x+\sqrt{1-2 / x}\right)\right] \\
& =-\left.\frac{d x}{d t}\right|_{\text {out }}
\end{aligned}
$$

for $t>0$. Thus, we can see that the trajectories of ingoing null geodesics, before the bounce, are the reflection of the trajectories of outgoing null geodesics after the bounce.

In the same way, we express the equation for the outgoing null geodesics before the bounce as:

$$
\begin{aligned}
\left.\frac{d x}{d t}\right|_{\text {out }} & =\frac{1}{\alpha} \sqrt{1-2 / x}\left(-\frac{2 \alpha}{3} \frac{t}{1+t^{2}} x+\sqrt{1-2 / x}\right) \\
& =-\frac{1}{\alpha}\left[\sqrt{1-2 / x}\left(\frac{2 \alpha}{3} \frac{t}{1+t^{2}} x-\sqrt{1-2 / x}\right)\right] \\
& =-\left.\frac{d x}{d t}\right|_{\text {in }}
\end{aligned}
$$

for $t>0$. We conclude that the congruence properties of outgoing null geodesics before the bounce are the same that for ingoing null geodesics after the bounce.

\section{SPACETIME STRUCTURE AFTER THE BOUNCE}

In order to prove that the surface $X_{-}=2, t=\infty$ is an event horizon the following conditions must be fulfilled: a) null ingoing radial geodesics reach $X_{-}=2, t=\infty$ in a finite interval of an affine parameter; b) the surface $X_{-}=2, t=\infty$ is regular.

To check if condition (a) is met, we make the following change of variables [15]:

$$
\begin{aligned}
& z=\sqrt{1-\frac{2}{x}}, \\
& l=\frac{1}{1+\alpha H(t)},
\end{aligned}
$$

where $0 \leq z \leq 1(2 \leq x<\infty)$ and $0<l<1$.

Since we are interested in the behavior of the geodesics for large values of $t$, we restrict the integration in terms of $l$ in the interval $0.75 \leq l<1$.

Given the expressions (24) and (25), the equations for 

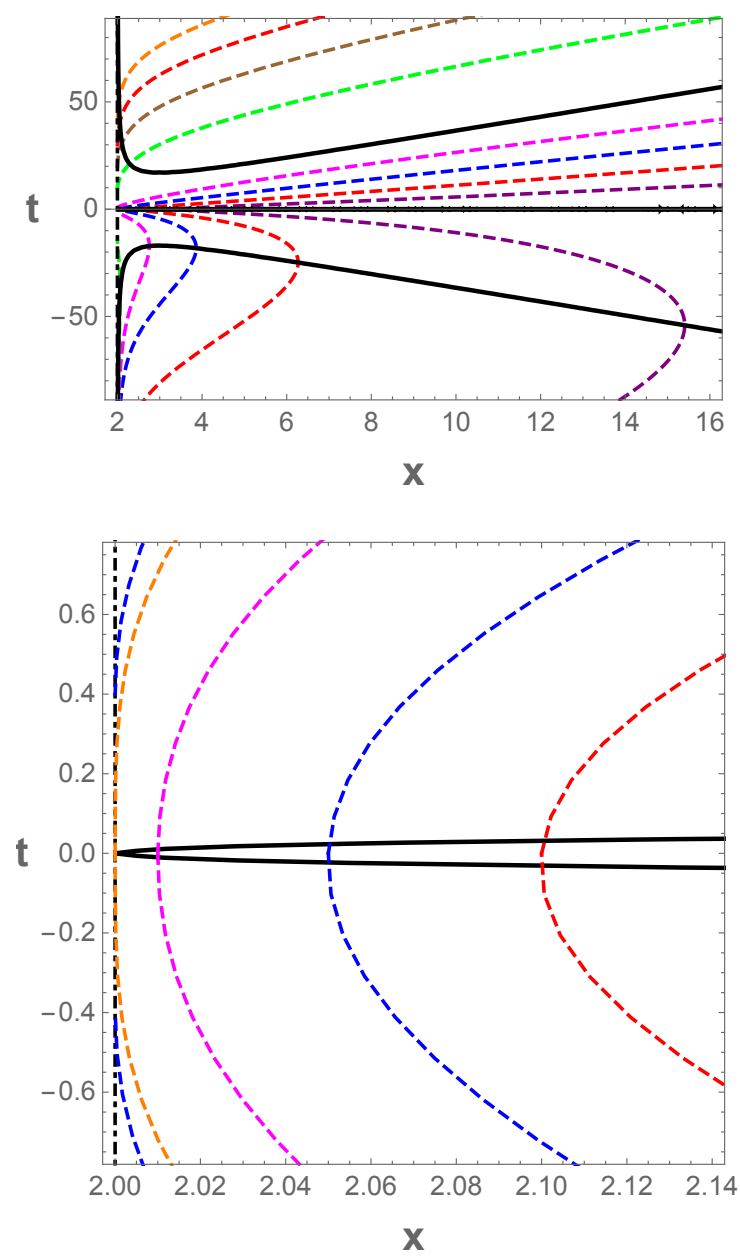

FIG. 9. Radial outgoing geodesics in McVittie spacetime for a bouncing cosmological background. The black line indicates the location of the trapping horizons while the dot dashed curve denotes the location of the singularity. The lower plot zooms into the region near the bounce. Here, $m_{0}=50 M_{\odot}$, and $t_{0}=5 \times 10^{-5} \mathrm{~s}$.

the ingoing geodesics 10 take the form
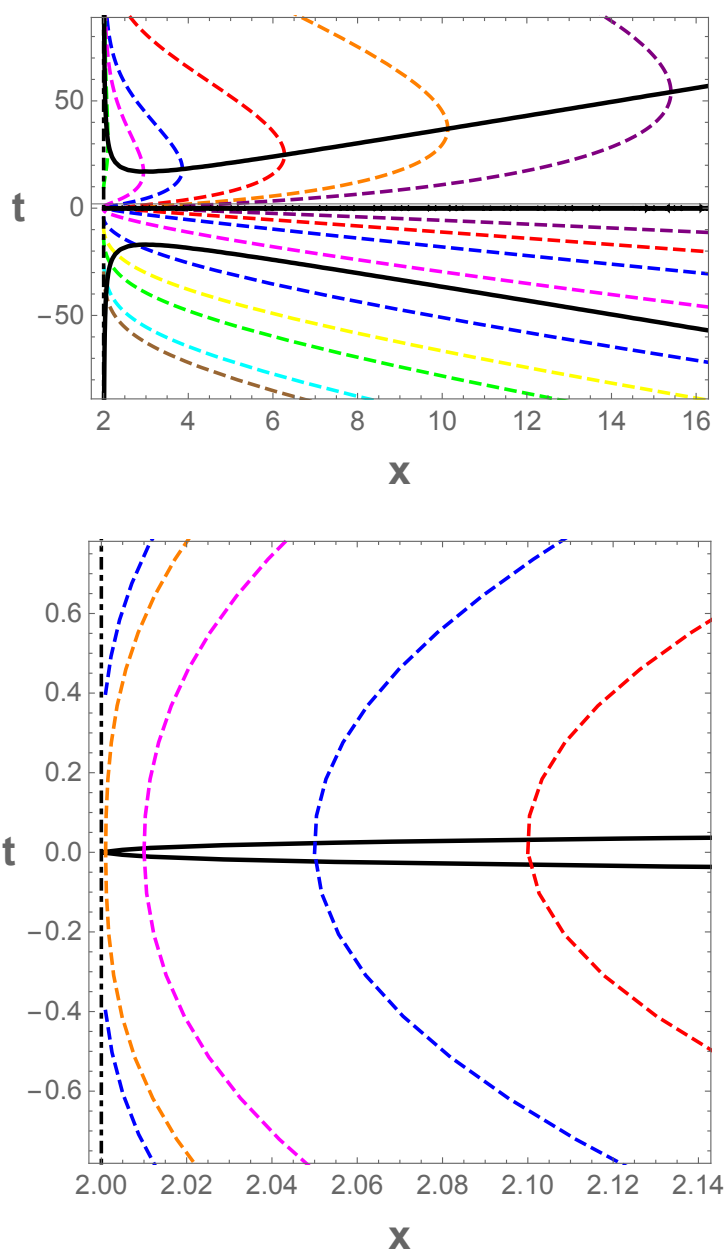

FIG. 10. Radial ingoing geodesics in McVittie spacetime for a bouncing cosmological background. The black line indicates the location of the trapping horizons while the dot dashed curve denotes the location of the singularity. The lower plot zooms into the region near the bounce. Here, $m_{0}=50 M_{\odot}$, and $t_{0}=5 \times 10^{-5} \mathrm{~s}$.

$$
\begin{aligned}
\frac{d z}{d l} & =\frac{\left(1-z^{2}\right)^{2}}{4}\left[\frac{2(1-l)}{l\left(1-z^{2}\right)}-z\right] \frac{d t}{d l}, \\
\frac{d l}{d t} & =\frac{9+l[-\beta+\alpha \sqrt{-9+\beta l}]}{3 l^{4}}, \\
\beta & =18+\left(\alpha^{2}-9\right) l .
\end{aligned}
$$




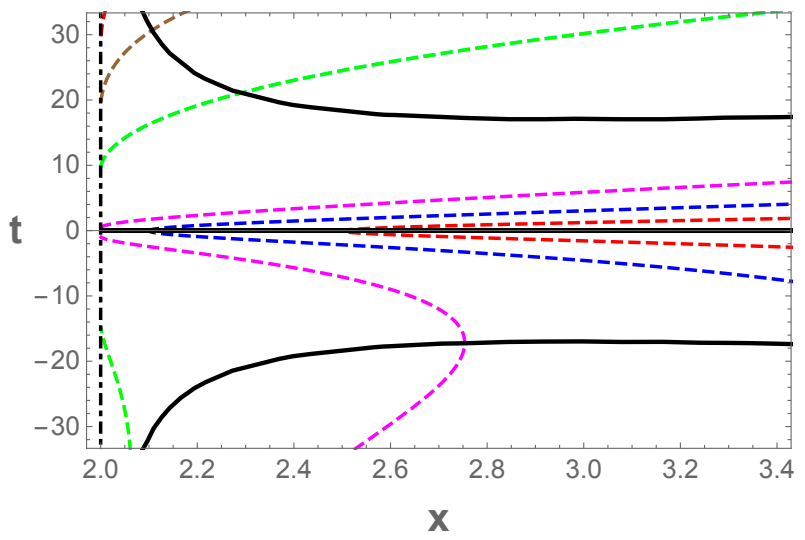

FIG. 11. Radial outgoing geodesics in McVittie spacetime for a bouncing cosmological background in the region close to the bounce. The black line indicates the location of the trapping horizons while the dot dashed curve denotes the location of the singularity. Here, $m_{0}=50 M_{\odot}$, and $t_{0}=5 \times 10^{-5} \mathrm{~s}$.

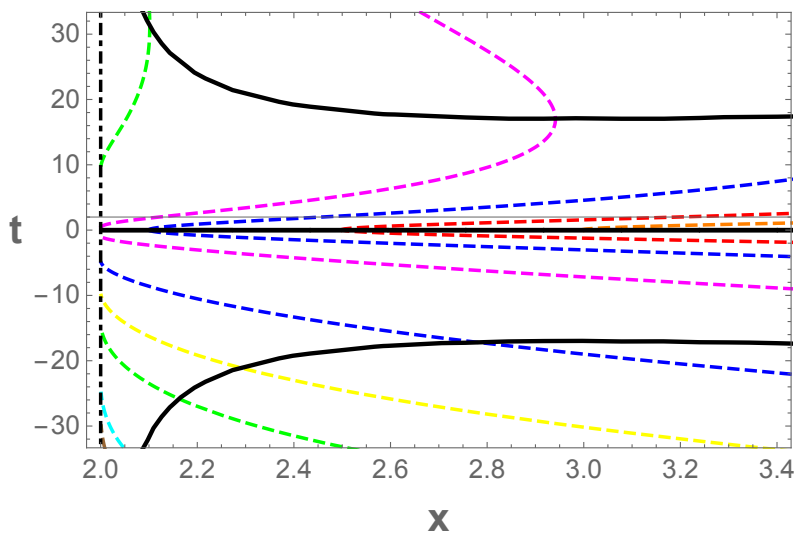

FIG. 12. Radial ingoing geodesics in McVittie spacetime for a bouncing cosmological background in the region close to the bounce. The black line indicates the location of the trapping horizons while the dot dashed curve denotes the location of the singularity. Here, $m_{0}=50 M_{\odot}$, and $t_{0}=5 \times 10^{-5} \mathrm{~s}$.

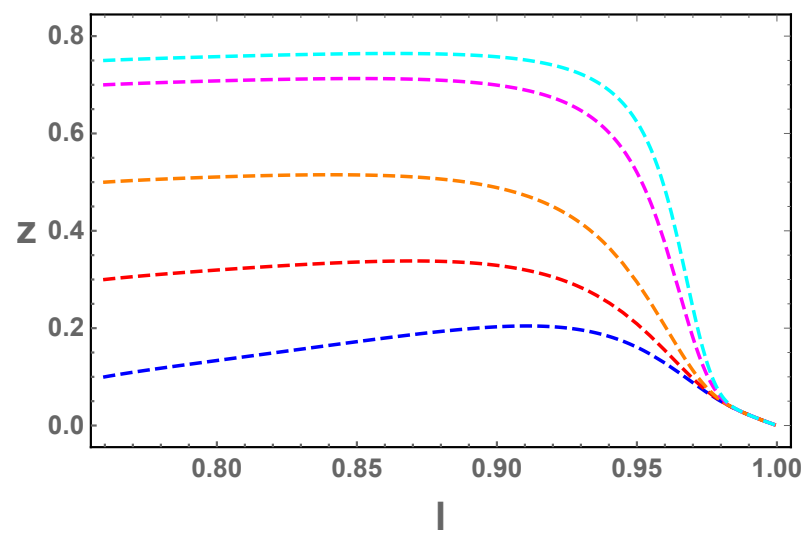

FIG. 13. Trajectories of radial ingoing null geodesics in the $z-l$ plane for McVittie spacetime in a bouncing cosmological model. Here, $m_{0}=50 M_{\odot}$, and $t_{0}=5 \times 10^{-5} \mathrm{~s}$.
In Figure 13, we plot the solution of the numerical integration of Eq. (26) for five different initial conditions. Clearly, radial ingoing null geodesics reach the surface $x=2(z=0)$ for $l=1$.

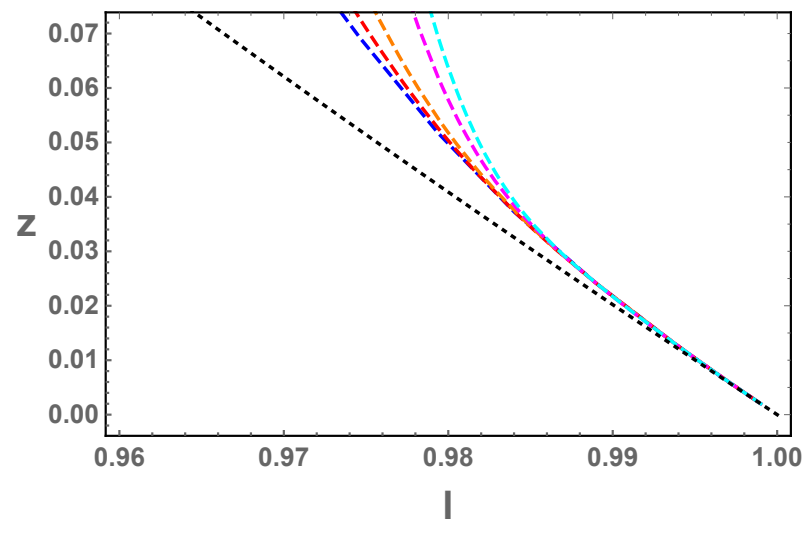

FIG. 14. Closeup in the $z-l$ plane showing the trajectories of radial ingoing null geodesics approaching the surface $x=$ 2. Expression (31) is represented by the dotted black curve. Here, $m_{0}=50 M_{\odot}$, and $t_{0}=5 \times 10^{-5} \mathrm{~s}$.

One way to decide whether condition (b), i.e. that $X_{-}$ is regular at large values of $t$, is valid or not is to calculate the components of the Riemann tensor using an appropriate Vierbein, and evaluate them on null geodesics approaching the surface. Since the model given by Eq.(13) behaves as a model dominated by dust for large $t$, namely

$$
H(t) \approx \frac{2}{3 t},
$$

we can use the result (valid for large values of $t$ ) obtained in [14]:

$$
t=\frac{2 x}{3 \sqrt{1-\frac{2}{x}}},
$$

or, in terms of the variables $z$ and $l$ :

$$
l z^{3}-l z-2 l+2=0 .
$$

Figure 14 shows that the asymptotic form of the null ingoing geodesics (given by Eq.(31)) describes extremely well the result of the exact numerical integration. Using GRTENSOR II [4], we have calculated the components of the Riemann tensor in the following Vierbein:

$$
\left[e^{j}{ }_{\mu}\right]=\left(\begin{array}{cccc}
\sqrt{H(t)^{2} r^{2}+f} & 0 & 0 & 0 \\
H(t) r & \frac{1}{\sqrt{1-\frac{2 m_{0}}{r}}} & 0 & 0 \\
0 & 0 & r & 0 \\
0 & 0 & 0 & r \sin \theta
\end{array}\right)
$$

The evaluation of the components on the null ingoing geodesics using (30) leads to finite values. Hence, the surface is regular. We have also evaluated, in the same 
limit, invariants built with first or second derivatives of tensors associated with curvature, such as $\left(\nabla_{\mu} R\right)\left(\nabla^{\mu} R\right)$ and $\left(\nabla_{\mu} \nabla_{\nu} R\right)\left(\nabla^{\mu} \nabla^{\nu} R\right)$, using RGTC 42. While invariants of the first kind yield a finite result, those of the second kind are divergent, in agreement with the result found in [14. Such a divergence does not influence the finiteness of the tidal forces, and is probably a consequence of the finite differentiability of the quantities describing the fluid 43 .

We conclude that the solution contains a black hole after the bounce.

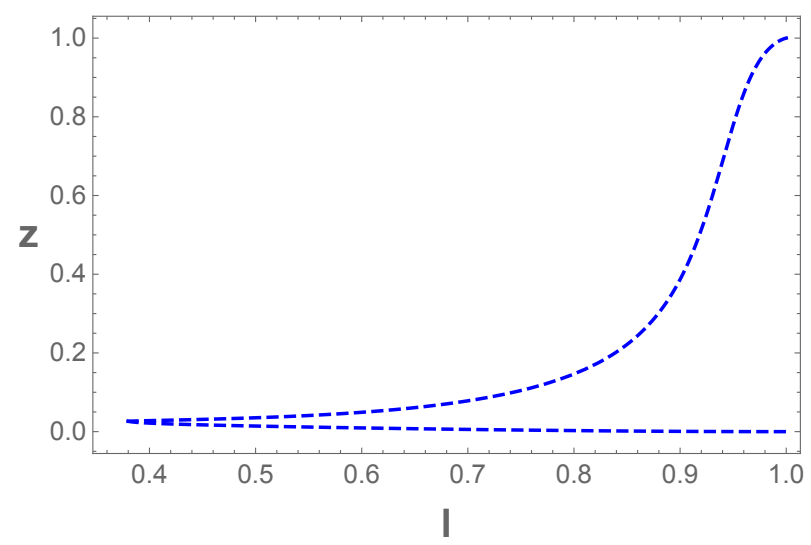

FIG. 15. Trajectory of a future-directed outgoing null geodesic that emerges from the singular point $(l, z)=(0,0)$ and reaches $z=1(x \rightarrow \infty)$ in a finite interval of time.

Using the change of variables given by Eqs. (24) and 25), we also integrate the trajectories of outgoing null geodesics. We show in Figure 15 the trajectory of a future-directed outgoing null geodesic that emerges from the singular point $(l, z)=(0,0)$, that is $(t, x)=(0,2)$ and reaches $z=1(x \rightarrow \infty)$ in a finite interval of time. Thus, we see that a naked singularity is present in the expanding phase up to the formation of the black hole.

\section{SPACETIME STRUCTURE BEFORE THE BOUNCE}

We showed in Figure 8 the existence of a trapped region, that is $\theta_{\text {in }} \theta_{\text {out }}>0$ where $\theta_{\text {in }}<0$ and $\theta_{\text {out }}<0$, for $t<0$. This region is bounded from below by inner $\left(X_{-}\right)$ and outer $\left(X_{+}\right)$trapping horizons for which $\theta_{\text {out }}=0$. As we approach the bounce, both horizons get closer and at

$$
t=t_{*}=-\left(\sqrt{3} \frac{G m_{0}}{c^{3} t_{0}}+\sqrt{\left(\sqrt{3} \frac{G m_{0}}{c^{3} t_{0}}\right)^{2}-1}\right),
$$

only a trapping horizon exist for $X_{-}=X_{+}=3$. If we choose $m_{0}=50 M_{\odot}$, and $t_{0}=5 \times 10^{-5} \mathrm{~s}$, then $t_{*}=-16.9$.

In Section IVA we proved that $X_{+}$is a null infinity $\left(X_{+} \rightarrow \infty\right.$ for $\left.t \rightarrow-\infty\right)$. The inner horizon $X_{-}$, defined

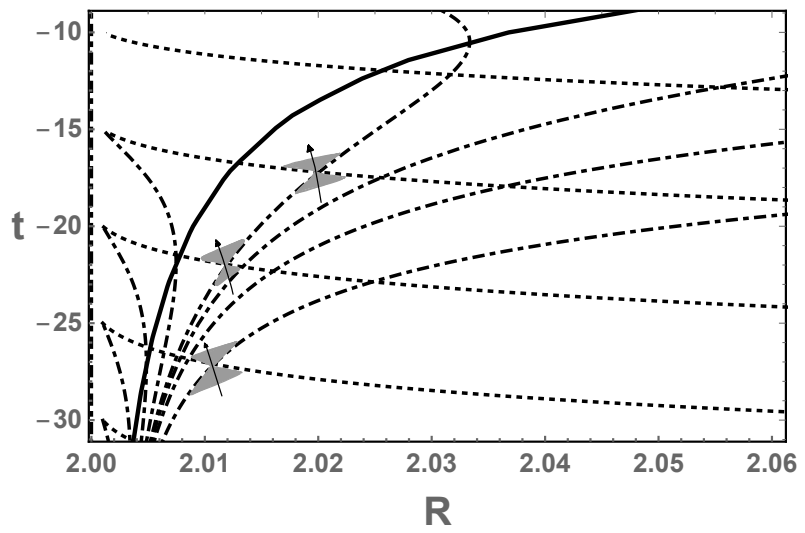

FIG. 16. Light cone structure in McVittie spacetime for a bouncing cosmological model in the region $t<0$. The dotted curves represent the null ingoing geodesics while the dot dashed curves the null outgoing geodesics. The regions shaded in grey show some light cones and the black arrow indicates the future direction.

for $2<X_{-} \leq 3$, covers the singularity and encloses a trapped region. We illustrate this situation in Figure 16 where the light cone structure for small and negative values of $t$ is plotted. The light cones have the trapping horizon in their local future . Both ingoing and outgoing null rays that cross $X_{-}$enter the trapped region and are unable to turn around and escape. This horizon, thus, acts as a one way membrane, hiding the singularity at $x=2$. This analysis leads us to conclude that in the time interval $-\infty<t<t_{*}$ the solution contains a black hole.

\section{DISCUSSION}

Given the analysis of the causal structure of the spacetime offered in the previous sections, we now provide a description of the evolution of the solution through cosmic time.

A black hole is present since the beginning of the contracting phase. The inner trapping horizon $X_{-}$increases its radius as the contraction gathers pace. The range of values of the radial coordinate for $X_{-}$is $2<X_{-} \leq 3$. Ingoing and outgoing null geodesics that cross the surface $X_{-}$, enter the trapped zone of the spacetime interior to the black hole. Close to the bounce, at $t_{*}$ (see Eq. (33)), the inner $X_{-}$and outer $X_{+}$trapping horizons merge and the black hole ceases to exist. This situation lasts for a short time. Trapping horizons appear again right before the bounce, and vanish right after it. These horizons are absent in other McVittie models (for instance, compare Figures 1 and 2 with 8 ). We associate these surfaces with the peculiarities of the cosmological background model, and more specifically to the presence of the bounce. There is no salient feature associated with these horizons: there, null geodesics just change their 


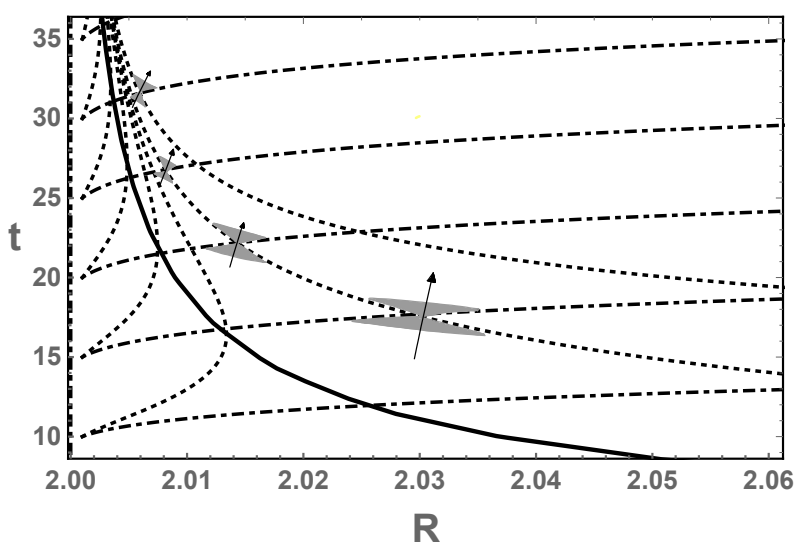

FIG. 17. Light cone structure in McVittie spacetime for a bouncing cosmological model in the region $t>0$. The dotted curves represent the null ingoing geodesics while the dot dashed curves the null outgoing geodesics. The grey shadow regions show some light cones and the black arrow indicates the future direction.

convergence properties.

Afterwards, the universe begins to expand and an inner $X_{-}$and outer $X_{+}$trapping horizons appear. For $t \rightarrow \infty$, the outer horizon becomes a FLRW null infinity, while $X_{-}$becomes an event horizon. Hence, as the universe expands a black hole starts to form. We show in Figure 17 the light cone structure for the spacetime after the bounce. We see that the surface $x=2$ is not in the local future of those light cones: in the process of black hole formation (the event horizon is not settled down) some geodesics are able to escape from the central source. As the universe expands, however, those outgoing geodesics that start at $x<X_{-}$have a smaller and smaller slope. In the limit $t \rightarrow \infty, d x / d t \rightarrow 0$ for outgoing geodesics, as computed from Eq. (10). This implies that outgoing null geodesics cannot leave the surface $x=2$ and the region contained by such boundary becomes trapped. In the distant future, the McVittie solution for a bouncing cosmological model harbors a black hole.

We show in Figures 18 and 19 qualitative Penrose diagrams of the McVittie spacetime for a bouncing cosmological model in the expanding and contracting phase, respectively. The dotted lines represent the trajectories of ingoing null geodesics and the dashed lines the trajectories of outgoing null geodesics. The black thick curve displays the trapping inner $\left(X_{-}\right)$and outer $\left(X_{+}\right)$horizons. The straight red line named $\mathcal{H}^{+}\left(\mathcal{H}^{-}\right)$represents $X_{-} \rightarrow 2, \quad t \rightarrow \infty\left(X_{-} \rightarrow 2, \quad t \rightarrow-\infty\right)$. There is a horizontal straight black line that characterises the region where the bounce occurs, that is $t=0$ and $x>2$. To the left, a dashed black and yellow line represents the singular surface $x=2, t$ finite. As usual, $\mathcal{J}^{+}\left(\mathcal{J}^{-}\right)$denotes the future (past) null infinity.

In the expanding phase, all ingoing geodesics, regardless of the initial conditions, reach the surface $\mathcal{H}^{+}$. Those outgoing null geodesics that start at the bounce extend to

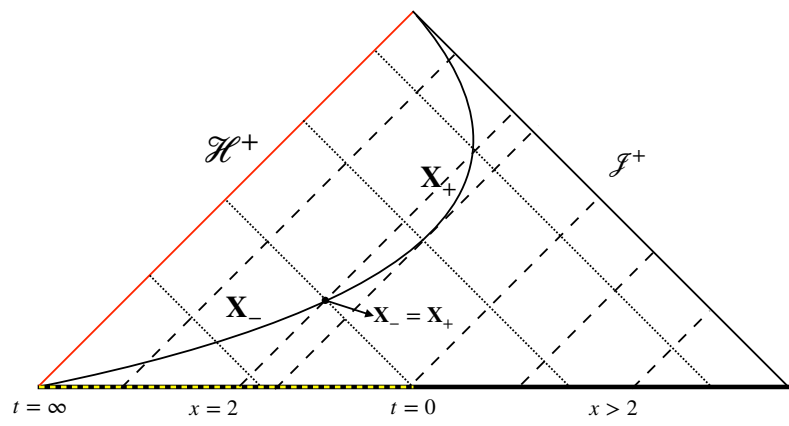

FIG. 18. Qualitative Penrose diagram of the McVittie spacetime for a bouncing cosmological model in the expanding region $(t>0)$. The dotted lines represent null ingoing geodesics while the dashed lines null outgoing geodesics. Here, $\mathcal{H}^{+}=X_{-} \rightarrow 2, \quad t \rightarrow \infty$, and $\mathcal{J}^{+}$is the future null infinity.

$\mathcal{J}^{+}$. There are also future directed outgoing radial null geodesics that emerge from the singular surface $x=2, t$ finite (this includes $(t, x)=(0,2))$. The latter evidences the existence of a spacelike naked singularity that lasts up to the formation of the black hole which corresponds to $\mathcal{H}^{+}$in Figure 18 .

As shown in Section IV A, in the contracting phase, ingoing and outgoing null geodesics reverse their character. Some ingoing geodesics end up in the singular surface while some others make it to the bounce. The same fate share outgoing null geodesics; all of them begin in $\mathcal{H}^{-}$. A black hole is present since the beginning of the contracting phase until $X_{-}=X_{+}$. The zone shaded in grey indicates the black hole region.

\section{CONCLUSIONS}

In this work the causal structure of McVittie spacetime for a bouncing cosmological background is analyzed. The location of the trapping horizons is computed, and the trajectories of null radial ingoing and outgoing null geodesics through cosmic time are obtained by numerical integration. A detailed study of the asymptotic behaviour of the metric is provided. Our main result is that the solution represents a dynamical black hole since the beginning of the contracting phase up to shortly before the bounce, and also in the distant future. Just before the bounce, the inner and outer trapping horizons merge and the black hole character of the solution is lost. After the bounce, the central inhomogeneity starts to act again, and for large and positive values of the cosmic time, a black hole is formed. Thus, we see that the global 


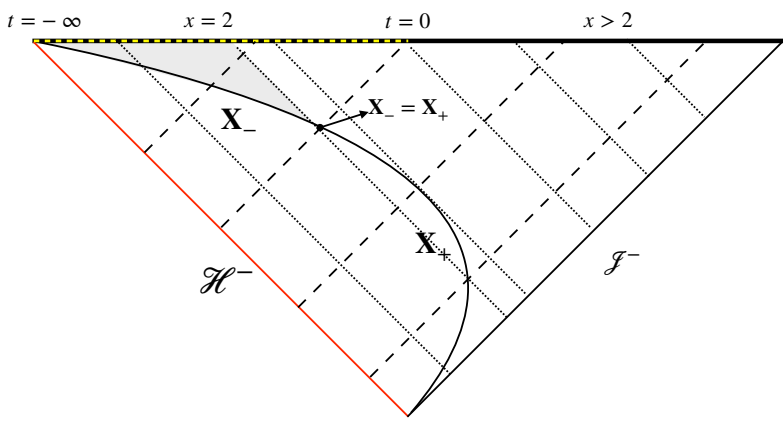

erature, there is no cosmological big singularity in the present metric. In fact, the solution admits trajectories that never encounter a singularity, that is, they are geodesically complete. This peculiar feature of the model is related to the occurrence of the bounce.

This work is a first step towards a better understanding of black holes embedded in a bouncing cosmological background; the current solution does not take into account the accretion of cosmological fluid by the central source. The Generalized McVittie metric naturally incorporates this effect by assuming an energy-momentum tensor of an imperfect fluid [40]. It remains an open issue whether the Generalized McVittie spacetime might contain a black hole for a bouncing cosmological background. We shall explore this issue in a future work.

\section{ACKNOWLEDGMENTS}

FIG. 19. Qualitative Penrose diagram of the McVittie spacetime for a bouncing cosmological model in the contracting region $(t<0)$. The dotted lines represent null ingoing geodesics while the dashed lines null outgoing geodesics. Here, $\mathcal{H}^{-}=X_{-} \rightarrow 2, \quad t \rightarrow-\infty$, and $\mathcal{J}^{-}$is the past null infinity.

dynamical state of the universe directly affects the conditions for the existence of black holes. In particular, for a contracting universe, black hole solutions are possible up to certain minimum scales.

Unlike all other McVittie models analyzed in the lit-
This work was supported by the Argentine agency CONICET (PIP 2014-00338) and the Spanish Ministerio de Ciencia e Innovacin (MICINN) under grant PID2019-105510GBC31 and through the Center of Excellence Mara de Maeztu 2020-2023 award to the ICCUB (CEX2019-000918-M). G.E.R. acknowledges support from the Coordenação de Aperfeiçoamento de Pessoal de Nivel Superior-Brasil (CAPES)-Codigo de Financiamento 001. Both D.P. and G.E.R. are very grateful to the Department of Physics of UERJ for kind hospitality. We are very grateful to two anonymous referees for their insightful comments.
[1] A. Borde, A. H. Guth, and A. Vilenkin, Inflationary Spacetimes Are Incomplete in Past Directions, Phys. Rev. Lett. 90, 151301 (2003), arXiv:gr-qc/0110012 [grqc].

[2] G. E. Romero, Adversus singularitates: The ontology of space-time singularities, Foundations of Science 18, 297 (2013).

[3] M. Novello and S. E. P. Bergliaffa, Bouncing Cosmologies, Phys. Rept. 463, 127 (2008), arXiv:0802.1634 [astro-ph]

[4] M. J. Rees, The collapse of the Universe: an eschatological study, The Observatory 89, 193 (1969).

[5] A. E. Sikkema and W. Israel, Black-hole mergers and mass inflation in a bouncing universe, Nature 349, 45 (1991)

[6] B. J. Carr and A. A. Coley, Persistence of Black Holes Through a Cosmological Bounce, International Journal of Modern Physics D 20, 2733 (2011), arXiv:1104.3796 [astro-ph.CO]

[7] T. Clifton, B. Carr, and A. Coley, Persistent black holes in bouncing cosmologies, Class. Quantum Grav. 34, 135005 (2017), arXiv:1701.05750 [gr-qc]].

[8] G. C. McVittie, The mass-particle in an expanding universe, Mon. Not. R. Astron. Soc. 93, 325 (1933)

[9] The areal radius coordinate $R$ is defined by $R:=\sqrt{\mathcal{A} / 4 \pi}$, where $\mathcal{A}$ is the area of the 2 -sphere of symmetry, and where

$$
d^{2} \Omega_{(2)}:=d \theta^{2}+\sin \theta^{2} d \phi^{2},
$$

is the line element on the unit 2-sphere [40].

[10] M. Carrera and D. Giulini, Generalization of McVittie's model for an inhomogeneity in a cosmological spacetime, Phys. Rev. D 81, 043521 (2010), arXiv:0908.3101 [gr-qc]

[11] B. C. Nolan, A point mass in an isotropic universe: Existence, uniqueness, and basic properties, Phys. Rev. D 58, 064006 (1998), arXiv:gr-qc/9805041 [gr-qc].

[12] B. C. Nolan, A point mass in an isotropic universe: II. Global properties, Class. Quantum Grav. 16, 1227 (1999).

[13] B. C. Nolan, A point mass in an isotropic universe: III. The region $\mathrm{R} \leq 2 \mathrm{~m}$, Class. Quantum Grav. 16, 3183 (1999), arXiv:gr-qc/9907018 [gr-qc].

[14] N. Kaloper, M. Kleban, and D. Martin, McVittie's legacy: Black holes in an expanding universe, Phys. Rev. D 81, 104044 (2010), arXiv:1003.4777 [hep-th],

[15] K. Lake and M. Abdelqader, More on McVittie's legacy: A Schwarzschild-de Sitter black and white hole embedded in an asymptotically $\Lambda$ CDM cosmology, Phys. Rev. D 84, 044045 (2011), arXiv:1106.3666 [gr-qc].

[16] F. J. Tipler, Singularities in conformally flat spacetimes, Physics Letters A 64, 8 (1977) 
[17] S. A. Hayward, General laws of black-hole dynamics, Phys. Rev. D 49, 6467 (1994), arXiv:gr-qc/9303006 [grqc].

[18] We are considering the minimal 6-parameter $\Lambda \mathrm{CDM}$ model; we assume that the curvature density parameter $\Omega_{k}=0$ and the equation of state parameter $w=-1$. The radiation density is neglected, $\Omega_{\mathrm{rad}}=0$. Thus $\Omega_{m}+\Omega_{\Lambda}=1$, being $\Lambda>0$.

[19] Lake and Abdelqader [15] proved that, in the case $H_{0}=$ $0, R=R_{-}=2 m_{0}$ for $t \rightarrow \infty$ is also a regular surface in the sense defined above.

[20] See Ref. 3] for a review.

[21] P. Peter and N. Pinto-Neto, Cosmology without inflation, Phys. Rev. D 78, 063506 (2008), arXiv:0809.2022 [gr-qc],

[22] F. T. Falciano, M. Lilley, and P. Peter, A Classical bounce: Constraints and consequences, Phys. Rev. D 77, 083513 (2008), arXiv:0802.1196 [gr-qc]].

[23] D. Wands, Cosmological perturbations through the big bang, Adv. Sci. Lett. 2, 194 (2009), arXiv:0809.4556 [astro-ph]

[24] A. Ijjas and P. J. Steinhardt, Classically stable nonsingular cosmological bounces, Phys. Rev. Lett. 117, 121304 (2016), arXiv:1606.08880 [gr-qc]

[25] O. Galkina, J. Fabris, F. Falciano, and N. Pinto-Neto, Regular Bouncing Solutions, Energy Conditions, and the Brans-Dicke Theory, JETP Lett. 110, 523 (2019), arXiv:1908.04258 [gr-qc]

[26] C. Almeida, H. Bergeron, J.-P. Gazeau, and A. Scardua, Three examples of quantum dynamics on the half-line with smooth bouncing, Annals Phys. 392, 206 (2018), 1708.06422 [quant-ph]

[27] A. P. Bacalhau, N. Pinto-Neto, and S. Dias Pinto Vitenti, Consistent Scalar and Tensor Perturbation Power Spectra in Single Fluid Matter Bounce with Dark Energy Era, Phys. Rev. D 97, 083517 (2018), arXiv:1706.08830 $[\mathrm{gr}-\mathrm{qc}]$.

[28] E. Frion and C. Almeida, Affine quantization of the Brans-Dicke theory: Smooth bouncing and the equivalence between the Einstein and Jordan frames, Phys. Rev. D 99, 023524 (2019), arXiv:1810.00707 [gr-qc]

[29] D. C. F. Celani, N. Pinto-Neto, and S. D. P. Vitenti,
Particle Creation in Bouncing Cosmologies, Phys. Rev. D 95, 023523 (2017), arXiv:1610.04933 [gr-qc]

[30] N. Pinto-Neto and J. Fabris, Quantum cosmology from the de Broglie-Bohm perspective, Class. Quant. Grav. 30, 143001 (2013), arXiv:1306.0820 [gr-qc]

[31] A. Ashtekar, T. Pawlowski, and P. Singh, Quantum nature of the big bang: Improved dynamics, Phys. Rev. D 74, 084003 (2006).

[32] V. Taveras, Corrections to the friedmann equations from loop quantum gravity for a universe with a free scalar field, Phys. Rev. D 78, 064072 (2008).

[33] H. Bergeron, A. Dapor, J. P. Gazeau, and P. Małkiewicz, Smooth big bounce from affine quantization, Phys. Rev. D 89, 083522 (2014)

[34] Limits on the parameters $a_{0}$ and $t_{0}$ can be found for instance in 38 .

[35] Notice that, contrary to the cases studied in 15], $H$ goes to zero at $t=0$.

[36] R. Nickalls, A new approach to solving the cubic: Cardans solution revealed, The Mathematical Gazette 77, 354359 (1993).

[37] Since we are now working with the radial dimensionless coordinate $x$, we denote the inner and outer radial coordinate of the trapping horizons as $X_{-} \equiv R_{-} /\left(G m_{0} / c^{2}\right)$ and $X_{+} \equiv R_{+} /\left(G m_{0} / c^{2}\right)$.

[38] E. Frion, N. Pinto-Neto, S. Vitenti, and S. Perez Bergliaffa, Primordial Magnetogenesis in a Bouncing Universe, Phys. Rev. D 101, 103503 (2020), arXiv:2004.07269 [grqc]

[39] B. Carr and F. Kühnel, Primordial Black Holes as Dark Matter: Recent Developments, Annual Review of Nuclear and Particle Science 70, annurev (2020), arXiv:2006.02838 [astro-ph.CO]

[40] V. Faraoni, ed., Lecture Notes in Physics, Berlin Springer Verlag, Lecture Notes in Physics, Berlin Springer Verlag, Vol. 907 (2015).

[41] Http://grtensor.phy.queensu.ca/.

[42] Http://www.inp.demokritos.gr/sbonano/RGTC/.

[43] P. Musgrave and K. Lake, Differential invariants and regularity, Classical and Quantum Gravity 12, L39 (1995). 